\title{
Coalition Formation and the Ancillary Benefits of Climate Policy
}

Michael Finus

Dirk T.G. Rübbelke

Stirling Economics Discussion Paper 2008-13

July 2008

Online at http://www.economics.stir.ac.uk 


\title{
Coalition Formation and the Ancillary Benefits of Climate Policy
}

\author{
Michael Finus* \\ Dirk T.G. Rübbelke**
}

* Corresponding author: Department of Economics, University of Stirling, Stirling FK9 4LA, Scotland, UK, email: michael.finus@stir.ac.uk, Tel: +44(0)1786467481, Fax: $+44(0) 1786467469$.

** Center for International Climate and Environmental Research - Oslo (CICERO), Pb. 1129 Blindern, 0318 Oslo, Norway, email: dirk.ruebbelke@cicero.uio.no

Acknowledgement: Dirk T.G. Rübbelke's research on this paper has been funded under the project "Integration of Climate and Air Quality Policies" sponsored by the Research Council of Norway and executed at the Center for International Climate and Environmental Research - Oslo. 


\title{
Coalition Formation and the Ancillary Benefits of Climate Policy
}

\begin{abstract}
Several studies found ancillary benefits of environmental policy to be of considerable size. These additional private benefits imply not only higher cooperative but also noncooperative abatement targets. However, beyond these largely undisputed important quantitative effects, there are qualitative and strategic implications associated with ancillary benefits: climate policy is no longer a pure but an impure public good. In this paper, we investigate these implications in a setting of non-cooperative coalition formation. In particular, we address the following questions. 1) Do ancillary benefits increase participation in international environmental agreements? 2) Do ancillary benefits raise the success of these treaties in welfare terms?
\end{abstract}

Keywords: ancillary benefits, climate policy, coalition formation, game theory, impure public goods

JEL classifications: C72, H87, Q54 


\section{Introduction}

International negotiations on climate change are now in the process of intensive search for a post-Kyoto protocol. The current situation is quite different from the 1990s when the Kyoto Protocol had been agreed upon by most industrialized countries. One important change is that China has meanwhile out-dated the US as the largest emitter of greenhouse gases (GHGs). Also the growing emissions of developing countries necessitate the inclusion of these countries in a post-Kyoto protocol if such an agreement shall be effective.

In particular developing countries have a vivid interest in combating global warming because it is especially their economies that will be negatively affected by climate change. Furthermore, there were significant extra benefits additional to slowing global warming which these countries could obtain if they actively contributed to climate protection. In this respect Aunan et al. (2007: 472) argue that a climate protection commitment would provide significant ancillary benefits to China ${ }^{1}$ since climate protection efforts will not only cause a reduction in GHG emissions but also reductions in particles- and $\mathrm{NO}_{\mathrm{X}}$-emissions. These (and other) non-GHG-emission reductions - in turn - will improve public health and will increase agricultural yields. Smith and Haigler (2008) recently offered sample calculations which illustrate the highdegree of ancillary benefit effectiveness for interventions in the household energy sector in developing countries. Further studies on ancillary benefits in the developing world are provided by, e.g. van Vuuren et al. (2003), Vennemo et al. (2006) for

1 Ancillary benefits are benefits generated by climate policy, but are not derived from the slowing of climate change. Other terms which convey this idea are secondary benefits, cobenefits and spillover benefits (see IPCC (2001)). The main difference between the terms is the relative emphasis given to benefits derived from slowing climate change versus other benefits. The types of impacts being covered are the same under each of these labels (Markandya and Rübbelke (2004: 489)). 
China, Dessus and O’Connor (2003) for Chile and Bussolo and O’Connor (2001) for India.

Although considered to be of less importance than in developing countries, ancillary benefits in industrialized countries may also be significant. Several studies found that ancillary benefits may even represent a multiple of primary benefits, i.e. the benefits derived from the slowing of climate change, ${ }^{2}$ as Pearce (2000: 523) illustrates in an overview. The compilation of ancillary benefit assessments for the US provided by Pearce (2000: 523) shows that ancillary benefits are a multiple of primary benefits, in the range between 0.07 and 6.67 (for European studies the range is between 0.98 (UK) and 6.93 (Germany)). An overview of further US studies is provided by Burtraw et al. (2003: 650-673).

As Ekins (1996a: 163, 1996b: 14) illustrates the inclusion of ancillary benefits in costbenefit analyses will cause a rise in optimal climate policy levels. Beyond the quantitative effect of ancillary benefits, there is also a qualitative dimension. Whereas the primary benefits of climate policy can be enjoyed globally, the ancillary benefits can only be enjoyed on a local or regional scale (IPCC (1996: 217)). ${ }^{3}$ In other words, climate protection generated by climate policy is a global public good, while the ancillary effects are mainly a private good from the climate policy providing country’s or region's point of view.

2 Rübbelke (2002: 5-23) provides an extensive distinction between primary and ancillary benefits.

3 Furthermore, in contrast to primary benefit, ancillary benefits arise almost immediately after the climate protection measure has been accomplished. Krupnick, Burtraw and Markandya (2000: 55) note: "we feel the heart of the analysis of ancillary benefits involves the here and now that is relevant to individual policy makers in a national context." 
Already Musgrave (1959: 13) stressed: “Certain public wants may fall on the border line between private and social wants, where the exclusion principle can be applied to part of the benefits gained but not to all.” Because of the joint-production property of climate policy and the fact that the jointly generated outputs are partly private to individual countries or regions and partly public to the whole world, climate policy is a so-called impure public good (see Cornes and Sandler (1996: 542)). It is important to note that the join-production property which we consider, i.e. implying that one strategy has two effects (a private and a public), differs from the analyses of mitigation versus adaptation (e.g. Barrett (2008)) or joint supply of climate mitigation and R\&D on new abatement technologies (e.g. Barrett (2006)), implying two strategies and two effects.

In recent years, the impure public good case also raised much attention in the theoretical oriented literature. Andreoni $(1989,1998)$ applies the basic approach developed by Cornes and Sandler (1984) to the field of philanthropy. Cornes and Sandler (1994) demonstrate that substitutability and complementarity of the private and public good lead to quite different comparative static results. Comparative static analyses based on the model by Cornes and Sandler (1994) are also conducted by Rübbelke (2003) who investigates climate policy as well as by Kotchen (2005) who considers environmentally friendly consumption. Rübbelke (2003) includes an alternative technology producing the impure public good's private characteristic independently of the public characteristic, while Kotchen (2005) allows for both, an independent production of the private as well as of the public characteristic.

In his analysis of green markets Kotchen (2006) also considers alternative technologies producing the impure public good's characteristics. He finds the surprising 
result that green technologies can be either welfare-improving or immiserizing, although they expand the consumer's choice possibilities and augment the production options. Kotchen and Moore (2007) employ the impure public good approach to the analysis of household participation in green-electricity programs. Dubin and Navarro (1988) address household refuse collection in an impure public good setting and Khanna, Huffman and Sandler (1994) analyze agricultural research.

Sandler and Murdoch (1990) provide an interesting study with a system of demand equations for pure and impure public good problems, distinguishing between NashCournot and Lindahl behaviour. They illustrate their technique with estimating military expenditure equations for a sample of ten NATO allies. They find that NashCournot, rather than Lindahl behaviour, best characterizes allies' behaviour. More importantly, they find that the impure public good specification provides a better fit than the pure public good specification of their model.

The impure public good property has not only distributional consequences, i.e. concerning who is enjoying the benefits, but has also consequences for the good's production prospects. "This follows because the jointly produced private output can serve a privatising role, not unlike the establishment of property rights” (Cornes and Sandler (1984: 595)). Nevertheless, impure public good provision tends to be suboptimal low because of the good's public characteristic (and despite its jointly generated private characteristic) and the associated free-rider incentives.

More specifically: on the one hand, ancillary benefits will call for higher globally optimal abatement levels. On the other hand, this will also be the case in the noncooperative equilibrium. For individual countries it is no longer irrelevant where the climate policy is accomplished. If a country does not contribute to climate protection, 
it will not enjoy ancillary benefits, though it can still benefit from the global benefits. Due to the impure public good nature of climate policy, international coordination is still needed. However, there is no global coercive authority which can enforce efficient global climate protection and consequently, countries have to voluntarily negotiate and agree upon such coordination.

It is therefore important to ascertain not only the largely undisputed quantitative and qualitative impacts of ancillary benefits for climate policy levels, but also their strategic implications. Recently, by using normal form games, Pittel and Rübbelke (2008) investigated the outcome of international negotiations on climate change in two different scenarios: one with and one without the inclusion of ancillary benefits. They find that if international negotiations are represented as a chicken game, ancillary benefits tend to have a positive influence on the propensity of countries to participate in an international agreement on climate change.

In this paper, we will investigate this idea further. We depart from the simple bimatrix games used in Pittel and Rübbelke (2008) and analyze the effects of ancillary benefits in the strategic setting of non-cooperative coalition formation. We consider three model versions, which have been considered in the literature. We reach quite different and mostly negative conclusions. The driving forces are different in the three models. In the first model, ancillary benefits lead to smaller coalition as they reduce the threshold of countries necessary for an agreement to be profitable. In the other two models, ancillary benefits increase not only abatement and payoffs of coalition members but also of outsiders, which leaves the free-rider incentives mainly unchanged. 
In the following, we develop our framework, analyze the size of stable coalitions and evaluate the success of coalition formation in welfare terms for three models in section 2. Finally, we summarize our main findings in section 3 and point to possible future research issues.

\section{The Model}

\subsection{Introduction}

We consider the two-stage cartel formation game with $N$ countries - which can be regarded as the working horse in the analysis of international environmental agreements since Barrett (1994), Carraro and Siniscalco (1993) and Hoel (1992). ${ }^{4}$ In the first stage, countries decide upon membership. Countries that join the coalition are called signatories (with superscript $M$ for member) and those that remain outside are called non-signatories (with superscript $N M$ for non-members) and assumed to act as singletons. Let $n \leq N$ denote the number of signatories with $N$ the total number of countries. In the second stage, signatories coordinate their action and choose their abatement by maximizing the aggregate payoff to all signatories. Non-signatories maximize their own payoff as singletons. The payoff function of an individual country is given by

(1) $\pi_{i}=B\left(\sum_{j=1}^{N} q_{j}\right)-C\left(q_{i}\right)$

where benefits, $B(X)$, depend on total abatement, $\sum_{j=1}^{N} q_{j}$, and $C(x)$ on individual abatement, $q_{i}$. We assume countries to be ex-ante symmetric, i.e. all have the same payoff function, but depending on whether they are signatories or non-signatories they

4 A survey is provided in Finus (2003, 2008). 
may receive different payoffs ex-post. ${ }^{5}$ Given the equilibrium abatement choices in the second stage, a signatory's payoff is denoted by $\pi_{i}^{M}(n)$ and a non-signatory payoff by $\pi_{i}^{N M}(n)$ in order to indicate that payoffs will depend on the number of signatories. Working back to the first stage, a coalition is said to be stable if it is internally and externally stable:

(2) Internal Stability: $\quad \pi_{i}^{M}(n) \geq \pi_{i}^{N M}(n-1) \forall i \in S$

(3) External Stability: $\quad \pi_{i}^{N M}(n)>\pi_{i}^{M}(n+1) \forall i \notin S$

denoting $S$ the set of coalition members and where we assume for convenience that in case a non-signatory is indifferent between joining and staying outside the coalition he will join. In some parts of the analysis it will be helpful to work with the stability function introduced by Hoel and Schneider (1997) $\Phi_{i}(n)=\pi_{i}^{M}(n)-\pi_{i}^{N M}(n-1)$ noticing that $\Phi_{i}(n) \geq 0$ means internal stability and $\Phi_{i}(n+1)<0$ external stability. In other words, if coalition $n$ is internally stable, then coalition $n-1$ is externally unstable and if coalition $n$ is externally stable, then coalition $n+1$ is internally unstable.

In case we consider ancillary benefits, payoff function (1) becomes:

(4) $\quad \pi_{i}=B\left(\sum_{j=1}^{N} q_{j}\right)+\alpha B\left(q_{i}\right)-C\left(q_{i}\right)$ with $\alpha \geq 0$.

Thus, the parameter $\alpha$ measures the weight which ancillary benefits receive in the payoff function of countries. These additional benefits only depend on the abatement conducted in this country. For simplicity, we assume the same functional form for

5 This assumption has been made frequently in the literature though it is certainly a simplification. See for instance Diamantoudi and Sartzetakis (2006) and Rubio and Ulph (2006). 
benefits from global abatement and ancillary benefits. The coalition game is solved in exactly the same way. However, payoffs will depend on $\alpha$ and hence we write $\pi_{i}^{M}(n, \alpha)$ and $\pi_{i}^{N M}(n, \alpha)$, respectively. Also stability is defined in the same way, accounting for the different notation, e.g. $\Phi_{i}(n, \alpha)=\pi_{i}^{M}(n, \alpha)-\pi_{i}^{N M}(n-1, \alpha)$ etc.

Subsequently, we consider specific benefit and cost functions that have been frequently used in the literature as analytical results for general payoff functions cannot be derived.

\subsection{Model with Linear Benefit and Cost Functions}

\subsubsection{No Ancillary Benefits}

The simplest payoff function assumes linear benefit and cost functions, which may almost be regarded as a toy model, though it has gained a lot of popularity in the recent literature (e.g. Barrett (2003), Ulph (2004) and Kolstad (2007)). The reason apart from its simplicity - is that this model version allows generating every stable coalition size which can be related to the benefit-cost ratio from abatement.

Consider the following payoff function where $b$ is a benefit and $c$ a cost parameter:

(5) $\quad \pi_{i}=b \sum_{j=1}^{N} q_{j}-c q_{i}$ with $b>0$ and $c>0$.

Since only the relation $b / c$ matters, we let $\gamma=b / c$ and rewrite (5):

(5)\# $\quad \pi_{i}=\gamma \sum_{j=1}^{N} q_{j}-q_{i}$ with $\gamma>0$.

In this simple model, players face only a binary choice: abate or not abate which we can normalize to $q_{i}=0$ and $q_{i}=1$. In order to make this model interesting for the 
subsequent analysis, we follow the standard assumption in the literature and assume that in the social optimum, abatement $q_{i}=1$ is an equilibrium choice, i.e.

(6) Ass. 1: $N \gamma-1>0$

and that in the Nash equilibrium no abatement $q_{i}=0$ is an equilibrium choice

(7) Ass. 2: $\gamma-1<0$.

That is abatement pays from a global but not from an individual perspective. Summarizing, this prisoners' type of incentive structure requires the following range of parameters:

(8) Ass. 1+2: $1>\gamma>1 / N$.

Total payoffs in the social optimum are $\Pi^{S}=N(N \gamma-1)$ and in the Nash equilibrium simply $\Pi^{N}=0$. If a coalition of $n$ countries forms, and if these signatories abate, they receive a payoff of $\pi_{i}^{M}(n)=n \gamma-1$ as for all $N-n$ non-signatories no abatement is a dominant strategy.

Suppose now one player leaves the coalition with $n$ signatories such that $n-1$ signatories are left. Consider first the possibility that signatories continue to abate (though the free-rider will no abate as this is the equilibrium choice as a singleton). Then the free-rider would receive a payoff of $\pi_{i}^{N M}(n-1)=(n-1) \gamma$. Internal stability would require that $\pi_{i}^{M}(n)=n \gamma-1 \geq(n-1) \gamma=\pi_{i}^{N M}(n-1)$ or $\gamma>1$ which we ruled out by assumption 2 in (7). Consider now the second possibility that signatories switch from abatement to no abatement once a signatory leaves and hence $\pi_{i}^{N M}(n-1)=0$. Let $n=n^{*}$, then internal stability requires 


$$
\pi_{i}^{M}(n)=n^{*} \gamma-1 \geq 0=\pi_{i}^{N M}\left(n^{*}-1\right) \text { and } \pi_{i}^{M}\left(n^{*}-1\right)=\left(n^{*}-1\right) \gamma-1<0 .
$$

In other words, $n^{*}$ is the largest integer of the relation $1 / \gamma$, i.e. $n^{*}=I(1 / \gamma)$. For any $n>n^{*}$, signatories would continue to abate after one signatory has left their coalition, which cannot be an equilibrium as argued above. For any $n<n^{*}$ signatories would not abate in the first place (as abatement is not profitable) and hence no coalition would form. It is easily checked that $n^{*}$ is also the only coalition which is externally stable and hence stable. Thus, in this model there is a strong threshold effect. The lower the benefit-cost ratio $\gamma$, the larger will be the threshold effect and hence $n^{*}$

Total payoff in the coalition equilibrium is given by $\Pi^{C}=n^{*} \pi_{i}^{M}\left(n^{*}\right)+\left(N-n^{*}\right) \pi_{i}^{N M}\left(n^{*}\right)$ and hence $\Pi^{C}=n^{*}(N \gamma-1)$ in this model. For comparison later, we measure the performance of this equilibrium in relative terms, which we call "closing the gap index “(Eyckmans and Finus (2006)) and define as follows:

$$
C G I:=\frac{\Pi^{C}-\Pi^{N}}{\Pi^{S}-\Pi^{N}}
$$

with $1 \geq C G I \geq 0$. For the model above, we find:

(11) $\quad C G I=\frac{n^{*}}{N}$.

Thus, anything else being equal, the closing the gap index decreases with the number of players involved in the externality problem - a result reflecting the public good nature of the global environmental problem and well-known from other models. 


\subsubsection{Ancillary Benefits}

Consider now ancillary benefits in this model. Since again only the relation $\gamma=b / c$ matters, the payoff function reads now:

$$
\pi_{i}(\alpha)=\gamma \sum_{j=1}^{N} q_{j}+\alpha \gamma q_{i}-q_{i} \text { with } \gamma>0 \text { and } \alpha \geq 0
$$

Like in the previous version, we have to impose some parameter restrictions in order to have an interesting incentive structure. Abatement is globally rational provided

(13) Ass. 3: $N \gamma+\alpha \gamma-1>0$

holds which gives us the lower bound of $\gamma$, i.e. $\gamma>1 /(N+\alpha)$. Abatement is not individually rational provided

(14) Ass. 4: $\gamma+\alpha \gamma-1<0$

holds which gives us the upper bound of $\gamma$, i.e. $\gamma<1 /(1+\alpha)$ and hence together we get:

(15) Ass. 3+4: $1 /(1+\alpha)>\gamma>1 /(N+\alpha)$.

Hence, compared to (8), the parameter range has to be smaller to preserve the prisoners' dilemma incentive structure. Note that if (14) does not hold, i.e. ancillary benefits would be very large, then all players would abate and Nash equilibrium and social optimum coincide. Consequently, there is no need for forming a coalition and therefore we disregard this possibility subsequently. Taken together, the global payoff in the social optimum is given by $\Pi^{S}(\alpha)=N(N \gamma+\alpha \gamma-1)$ and in the Nash equilibrium by $\Pi^{N}(\alpha)=0$. 
Following exactly the same arguments presented above, it turns out that the equilibrium coalition $n^{*}(\alpha)$ is the coalition for which (see (9) above)

$$
\begin{aligned}
& \pi_{i}^{M}\left(n^{*}, \alpha\right)=n^{*}(\alpha) \gamma+\alpha \gamma-1 \geq 0=\pi_{i}^{N}\left(n^{*}-1, \alpha\right) \text { and } \\
& \pi_{i}^{M}\left(n^{*}-1, \alpha\right)=\left(n^{*}(\alpha)-1\right) \gamma+\alpha \gamma-1<0
\end{aligned}
$$

holds and hence $n^{*}(\alpha)=I((1 / \gamma)-\alpha)$. Since $n^{*}(\alpha)=I((1 / \gamma)-\alpha) \leq I(1 / \gamma)=n^{*}$, ancillary benefits will lead to the same or a smaller coalition than without ancillary benefits. In particular, the larger ancillary benefits are, measured by the parameter $\alpha$, the smaller will be the equilibrium coalition. The reason is that ancillary benefits reduce the threshold number of signatories for which it pays the coalition members to abate and hence to form a coalition at all.

Certainly the size of a coalition is one indicator of success. However, the global payoff is what counts. The global coalition payoff is given by $\Pi^{C}(\alpha)=n^{*}(\alpha)(N \gamma+\alpha \gamma-1)$. Since payoff functions without and with ancillary benefits are different, we again measure the success of the coalition equilibrium in relative terms (see (10) above) and find:

$$
\operatorname{CGI}(\alpha)=\frac{n^{*}(\alpha)}{N}
$$

Since $n^{*} \geq n^{*}(\alpha)$, and comparing (11) with (17), it is obvious that ancillary benefits cannot improve, but can only worsen the situation. As we will see, this negative conclusion will not change much in the subsequent models. 


\subsection{Model with Linear Benefit and Quadratic Cost Functions}

\subsubsection{No Ancillary Benefits}

Consider the following payoff function which has been considered for instance in Botteon and Carraro (1997), Barrett (2006) and Finus and Maus (2008):

$$
\pi_{i}=b \sum_{j=1}^{N} q_{j}-\frac{c}{2} q_{i}^{2} \text { with } b>0 \text { and } c>0 \text {. }
$$

Also for this model only the relation $b / c$ matters, and hence we let $\gamma=b / c$ and rewrite (18):

(18)\# $\quad \pi_{i}=\gamma \sum_{j=1}^{N} q_{j}-\frac{1}{2} q_{i}^{2}$ with $\gamma>0$.

The first order conditions of signatories read $n \gamma-q_{i}^{M}=0$ and for non-signatories $\gamma-q_{i}^{N M}=0$, assuming an interior solution and denoting abatement of signatories with superscript $M$ (members) and abatement of non-signatories with superscript $N M$ (non-members). Hence, $q_{i}^{M}(n)=n \gamma$ and $q_{i}^{N M}=\gamma$ and total abatement is given $Q^{C}(n)=n^{2} \gamma+(N-n) \gamma$. If one coalition member leaves, $q_{i}^{M}(n-1)=(n-1) \gamma$, $q_{i}^{N M}=\gamma$ and $Q^{C}(n-1)=(n-1)^{2} \gamma+(N-n+1) \gamma$. Substitution into the payoff function gives

$$
\begin{aligned}
& \pi_{i}^{M}(n)=\frac{1}{2} \gamma^{2}\left(n^{2}+4 N+1-2 n\right), \pi_{i}^{N M}(n)=\gamma^{2}\left(n^{2}+2 N-n\right), \\
& \pi_{i}^{N M}(n-1)=\gamma^{2}\left((n-1)^{2}+2 N-(n-1)\right)
\end{aligned}
$$

Computation of the stability function $\Phi_{i}(n)=\pi_{i}^{M}(n)-\pi_{i}^{N M}(n-1)$ shows that $\Phi_{i}(n) \geq 0$ for $n \in\{2,3\}$ and $\Phi_{i}(n+1)<0$ for $n \geq 3$ and hence $n^{*}=3$. We can now 
compute the global payoff in the coalition equilibrium by using $n^{*}=3$ : $\Pi^{C}=n^{*} \pi_{i}^{M}\left(n^{*}\right)+\left(N-n^{*}\right) \pi_{i}^{N M}(n)$. Moreover, the global payoff in the social optimum is simply $N$ times $\pi_{i}^{M}(n)$ by substituting $N$ for $n$ in $\pi_{i}^{M}(n)$. Similarly, the global payoff in the Nash equilibrium is simply $N$ times $\pi_{i}^{N M}(n)$ by setting $n=1$ in $\pi_{i}^{N M}(n)$. We find for the closing the gap index:

(20) $\quad C G I=\frac{12(N-2)}{N(N-1)^{2}}$

which is positive but smaller than 1 if $N>3$ because then the grand coalition is not stable $\left(n^{*}<N\right)$. Again, it straightforward to show that $\partial C G I / \partial N<0$ provided $N>3$. Hence, as in the first model (section 2.2), the coalition improves upon the noncooperative outcome but achieves only little if the number of countries is large which suffer from the externality.

\subsubsection{Ancillary Benefits}

Modifying payoff function (18)\# to account for ancillary benefits gives:

(21) $\pi_{i}=\gamma \sum_{j=1}^{N} q_{j}+\alpha \gamma q_{i}-\frac{1}{2} q_{i}^{2}$ with $\gamma>0$ and $\alpha \geq 0$.

Now, $q_{i}^{M}(n, \alpha)=\gamma(n+\alpha)$ and $q_{i}^{N M}(\alpha)=\gamma(1+\alpha)$. Hence, signatories and nonsignatories adjust their abatement levels upward. This is also the case in the social optimum and the Nash equilibrium with $q_{i}^{S}(\alpha)=\gamma(N+\alpha)$ and $q_{i}^{N}(\alpha)=\gamma(1+\alpha)$, respectively. Again, we can compute $\pi_{i}^{M}(n, \alpha), \pi_{i}^{M N}(n, \alpha)$ and $\pi_{i}^{M N}(n-1, \alpha)$ now accounting for ancillary benefits. The stability check delivers the same coalition size, $n^{*}(\alpha)=3$ and the closing the gap index is exactly (20). Roughly speaking, all rela- 
tions just change by the factor $\alpha$. The drop in benefits (benefits from global abatement and ancillary benefits) and the drop in abatement costs when leaving a coalition become larger, but by the same factor. Hence, stability is not affected. The same applies when computing relative performance. Consequently, compared to the previous model, ancillary benefits have at least not a negative effect, but the conclusion is also not brighter.

\subsection{Model with Quadratic Benefit and Quadratic Cost Functions}

Consider the following payoff function:

$$
\pi_{i}=b\left(a \sum_{j=1}^{N} q_{j}-\frac{1}{2} \sum_{j=1}^{N} q_{j}^{2}\right)+\alpha b\left(a q_{i}-\frac{1}{2} q_{i}^{2}\right)-\frac{c}{2} q_{i}^{2} \text { with } b>0, c>0 \text { and }
$$

$$
\alpha \geq 0
$$

This payoff function is essentially the same as in Barrett (1994), Diamantoudi and Sartzetakis (2006) and Rubio and Ulph (2006), though they assume $\alpha=0 .^{6}$ In order to save space, we derive in this section the case without and with ancillary benefits together, noticing from the previous analysis that no ancillary benefits is a special case of ancillary benefits when setting $\alpha=0$. Since this model is far more messy in terms of derivations than the previous models, we sketch here only the main arguments of the analysis and relegate the details to the appendix.

The first thing to notice is that - inserting equilibrium abatement levels into the marginal benefit function from global abatement, marginal benefits could become negative. This is equivalent to saying that total abatement in the social optimum could

$6 \quad$ Diamantoudi and Sartzetakis (2006) and Rubio and Ulph (2006) specify their model in terms of emissions but the incentive structure remains the same as long as non-negativity constraints are observed (which we do) as this authors prove. However, different from these authors we do not assume Stackelberg leadership of signatories to be consistent with the previous models where Stackelberg leadership would make no difference due to dominant strategies. See Finus (2003). 
be smaller than in the Nash equilibrium if the weight $\alpha$ which ancillary benefits receive is sufficiently large. In the appendix we show that a sufficient condition to rule this out and to ensure reasonable results, is:

(23) Ass. 5: $\frac{1}{(N-1) \gamma} \geq \alpha$ with $\gamma=b / c$.

In a next step we analyze the stability function, which we write $\Phi_{i}(n, \Gamma)=\pi_{i}^{M}(n, \Gamma)-\pi_{i}^{N M}(n-1, \Gamma)$ with $\Gamma$ a set of parameters, comprising $\alpha$, $\gamma=b / c$ and $N$. It turns out that stability does not depend on the parameter $a$. Not surprising for $N=2, \Phi_{i}(2, \Gamma)>0$, i.e. the grand coalition is stable. In order to analyze the more interesting case of $N \geq 3$, we first show that $\partial \Phi_{i}(n, \Gamma) / \partial N<0$ (and $\left.\partial^{2} \Phi_{i}(n, \Gamma) / \partial N^{2}<0\right)$. That is, with an increasing number of coalition members, it becomes more difficult to establish stability for a given number of signatories, $n$. Hence, in order to test whether $\Phi_{i}(n, \Gamma)$ can be positive for $n>2$, we substitute $N=3$ and $n=3$ into the stability function and find $\Phi_{i}(3, \Gamma)<0$. Hence, we can conclude that there cannot be a stable coalition larger than 2. This finding is well known and probably goes back to Carraro and Siniscalco (1991). The new and interesting part is that this also holds for ancillary benefits for any $\alpha$ defined in the range in (23).

In a next step, we set $\alpha=0$ and $n=2$ in the stability function but make no further assumptions, i.e. $\Phi_{i}(n=2, \alpha=0, \gamma, N)$. We establish that for $0 \leq \gamma \leq \bar{\gamma}(N) \leq 1$ $\Phi_{i}(n=2, \alpha=0, \gamma, N) \geq 0$ is positive where $\bar{\gamma}(N)$ is an upper bound which decreases in $N, \partial \bar{\gamma}(N) / \partial N<0$. That is, with an increasing number of players $N$, the range 
of the parameter $\gamma$ for which internal stability can be guaranteed for two signatories decreases.

Now we let $\alpha \geq 0$ in the stability function, $\Phi_{i}(n=2, \alpha, \gamma, N)$, and show that $\Phi_{i}(n=2, \alpha, \gamma, N) \geq 0$ if $\alpha>\Psi(\gamma, N)$. Since $\alpha$ is bounded from above as spelled out in (23), we get:

$$
\frac{1}{(N-1) \gamma} \geq \alpha \geq \Psi(\gamma, N) \text { with } \gamma=b / c
$$

such that ancillary benefits can make a difference. For $\gamma=\bar{\gamma}(N)$ it can be shown that there exists an $\alpha$ satisfying this inequality. However, the upper bound of $\alpha$ in (24), $1 /((N-1) \gamma)$, decreases in $\gamma$ and $N$ and the lower bound of $\alpha, \Psi(\gamma, N)$, increases in $\gamma$ and $N$. Thus, for a given $N$, we can only slightly increase $\gamma$ above $\bar{\gamma}(N)$ such that $\alpha$ can still satisfy condition (24). With increasing $N$ this possibility diminishes further.

For instance, denoting the upper bound of $\gamma$ with ancillary benefits by $\bar{\gamma}(N, \alpha)$, $\bar{\gamma}(N, \alpha)>\bar{\gamma}(N)$, such that for $0 \leq \gamma \leq \bar{\gamma}(N, \alpha) \Phi_{i}(n=2, \alpha, \gamma, N) \geq 0$, then we find for $N=3: \bar{\gamma}(N, \alpha)=0.59$ but only $\bar{\gamma}(N)=0.41$ without ancillary benefits. However, already for $N=7: \bar{\gamma}(N, \alpha)=0.08$, which is only slightly above $\bar{\gamma}(N)=0.07$ without ancillary benefits. Thus, the possible extension of the parameter range of $\gamma$ for which a coalition of two players is stable with ancillary benefits tends to zero for a sufficiently large number of players.

Even more interesting is that one can show that for $N \geq 4$, the closing the gap index, CGI, as defined in (10), decreases in $\alpha$ for $0 \leq \gamma \leq \bar{\gamma}(N) \leq 1$. This is the parameter 
range for which a two player coalition would be stable even without ancillary benefits. Thus, it is only for $\bar{\gamma}(N)<\gamma \leq \bar{\gamma}(N, \alpha)$ where no coalition would be stable without ancillary benefits and hence $C G I=0$ where ancillary benefits can make a difference $(C G I>0)$. However, as pointed out above, with increasing $N$ the difference between $\bar{\gamma}(N, \alpha)$ and $\bar{\gamma}(N)$ shrinks and is almost zero already for $N=10$.

Taken together for this model the effect of ancillary benefits is mixed, though also not very encouraging. First of all, ancillary benefits cannot expand the coalition to more than 2 signatories which is the upper limit without ancillary benefits. Second, for the parameter range of the benefit-cost parameter $\gamma$ for which a coalition of two signatories is stable without ancillary benefits, an increasing weight $\alpha$ of ancillary benefits has a negative impact on the relative performance measured as the closing the gap index. Third, in a few instances, ancillary benefits can expand the range of the benefit-cost parameter for which a two player coalition is stable. This expansion is negligible if the total number of players is sufficiently large.

\section{Summary and Conclusions}

Ancillary benefits have attracted much attention in climate change research and are regularly found to be of significant size. The IPCC (2007: 623) stresses the importance of ancillary benefits in the design of air pollution reduction and energy security policy and the rise in rural employment. The “efficiency-raising impact” of an inclusion of ancillary benefits in cost-benefit analyses has been highlighted recurrently. Estimated efficient climate protection levels tend to rise considerably due to the inclusion of ancillary benefits. 
However, what was largely omitted in the scientific literature is the analysis of the strategic implications of the privatizing effect of ancillary benefits. Intuitively, one would expect that taking account of ancillary benefits will alleviate free-rider incentives and hence will raise the attractiveness of participation in international agreements to slow climate change.

In our paper, we focused on the strategic implications of ancillary benefits. We analysed the impacts of ancillary benefits in the strategic setting of non-cooperative coalition formation. Our results were counter-intuitive - at least at first thought. We found that ancillary benefits have a neutral or negative impact on the size of stable coalitions the success of coalition formation measured in welfare terms. Only in the model with quadratic benefit and quadratic cost functions could ancillary benefits expand the range of the benefit-cost parameter for which a two player coalition can be stable, though this occurs only in a few instances. However even then the expansion was insignificant if the total number of players is sufficiently large.

The conclusion which can be drawn from these results is that although ancillary benefits provide additional incentives to protect the climate, they will not raise the likelihood of an efficient global agreement on climate change to come about.

The intuition behind this result is that countries taking the private ancillary benefits to a greater extent into account will provide more climate policy, irrespective of an international agreement. The relative importance of an international agreement for climate protection is reduced since the privatizing effect of ancillary benefits already provides incentives for protection in a non-cooperative setting. Hence, though ancillary benefits provide an additional incentive to participate and to raise abatement contributions, they also provide an additional incentive to leave the agreement. 
As outlined in the introduction, countries are enjoying different levels of ancillary benefits. In developing countries these seem to be more important than in industrialized countries. This heterogeneity should be included in future models. Furthermore, it should be taken into account that ancillary effects, like the mitigation of local air pollution, can also be produced by technologies (like desulphurisation installations) which do not produce climate protection. These technologies tend to reduce the level of ancillary benefits. Moreover, technological interdependencies should be explored in future research. Finally, there may also be ancillary cost, e.g. induced by a reduction in energy self-sufficiency, associated with certain climate policies. It would be interesting to see how these affect the outcome. 


\section{References}

Andreoni, J. (1989), Giving with Impure Altruism: Applications to Charity and Ricardian Equivalence. Journal of Political Economy, 97, 1447-1458.

Andreoni, J. (1998), Toward a Theory of Charitable Fund-raising. Journal of Political Economy, 106, 1186-1213.

Aunan, K., Berntsen, T., O’Connor, D., Hindman Persson, T., Vennemo, H. and Zhai, F. (2007), Benefits and Costs to China of a Climate Policy. Environment and Development Economics, 12, 471-497.

Barrett, S. (1994), Self-enforcing International Environmental Agreements. Oxford Economic Papers, 46, 878-894.

Barrett, S. (2003), Environment and Statecraft: The Strategy of Environmental Treaty-making. Oxford University Press, New York.

Barrett, S. (2006), Climate Treaties and “Breakthrough" Technologies. American Economic Review, 96, 22-25.

Barrett, S. (2008), Dikes v. Windmills: Climate Treaties and Adaptation, paper presented at the workshop "The Environment, Technology and Uncertainty" of the Ragnar Frisch Centre for Economic Research, Oslo.

Botteon, M. and Carraro, C. (1997), Burden-sharing and Coalition Stability in Environmental Negotiations with Asymmetric Countries. In: Carraro, C. (ed.), International Environmental Negotiations: Strategic Policy Issues. Edward Elgar, Cheltenham, ch. 3, 26-55.

Burtraw, D., Krupnick, A., Palmer, K., Paul, A., Toman, M. and Bloyd, C. (2003), Ancillary Benefits of Reduced Air Pollution in the U.S. from Moderate Greenhouse Gas Mitigation Policies in the Electricity Sector. Journal of Environmental Economics and Management, 45, 650-673.

Bussolo, M. and O'Connor, D. (2001), Clearing the Air in India: The Economics of Climate Policy with Ancillary Benefits. Working Paper No. 182, OECD Development Centre, Paris.

Carraro, C. and Siniscalco, D. (1991), Strategies for the International Protection of the Environment. Working Paper, March 1991, Fondazione Eni Enrico Mattei, Milano.

Carraro, C. and Siniscalco, D. (1993), Strategies for the International Protection of the Environment. Journal of Public Economics, 52, 309-328.

Cornes, R.C. and Sandler, T. (1984), Easy Riders, Joint Production, and Public Goods. Economic Journal, 94, 580-598. 
Cornes, R.C. and Sandler, T. (1994), The Comparative Static Properties of the Impure Public Good Model. Journal of Public Economics, 54, 403-421.

Cornes, R.C. and Sandler, T. (1996), The Theory of Externalities, Public Goods and Club Goods, Cambridge University Press, Cambridge.

Dessus, S. and O’Connor, D. (2003), Climate Policy without Tears: CGE-based Ancillary Benefits Estimates for Chile. Environmental and Resource Economics, 25, 287-317.

Diamantoudi E. and Sartzetakis, E. (2006), Stable International Environmental Agreements: an Analytical Approach. Journal of Public Economic Theory, 8, 247263.

Dubin, J.A. and Navarro, P. (1988), How Markets for Impure Public Goods Organize: The Case of Household Refuse Collection. Journal of Law, Economics, and Organization, 4, 217-242.

Ekins, P. (1996a), How Large a Carbon Tax is Justified by the Secondary Benefits of $\mathrm{CO}_{2}$ Abatement?. Resource and Energy Economics, 18, 161-187.

Ekins, P. (1996b), The Secondary Benefits of $\mathrm{CO}_{2}$ Abatement: How Much Emission Reduction Do They Justify?. Ecological Economics, 16, 13-24.

Eyckmans, J. and Finus, M. (2006), Coalition Formation in a Global Warming Game: How the Design of Protocols Affects the Success of Environmental Treaty-making. Natural Resource Modeling, 19, 323-358.

Finus, M. (2003), Stability and Design of International Environmental Agreements: The Case of Global and Transboundary Pollution. In: Folmer, H. and T. Tietenberg (eds.), International Yearbook of Environmental and Resource Economics 2003/4. Edward Elgar, Cheltenham, ch. 3, 82-158.

Finus, M. (2008), Game Theoretic Research on the Design of International Environmental Agreements: Insights, Critical Remarks and Future Challenges. International Review of Environmental and Resource Economics, 2, 1-39.

Finus, M. and Maus, S. (2008), Modesty May Pay. Journal of Public Economic Theory, forthcoming.

Hoel, M. (1992), International Environment Conventions: The Case of Uniform Reductions of Emissions. Environmental and Resource Economics, 2, 141-159.

Hoel, M. and Schneider, K. (1997), Incentives to Participate in an International Environmental Agreement. Environmental and Resource Economics, 9, 153-170.

IPCC (1996), Climate Change 1995 - Economic and Social Dimensions of Climate Change. Cambridge University Press, Cambridge. 
IPCC (2001), Climate Change 2001 - Mitigation. Cambridge University Press, Cambridge.

IPCC (2007), Climate Change 2007 - Mitigation of Climate Change. Cambridge University Press, Cambridge.

Khanna, J., Huffman, W. and Sandler, T. (1994), Agricultural Research Expenditures in the U.S.: a Public Goods Perspective. Review of Economics and Statistics, 76, 267-277.

Kolstad, C. (2007), Systematic Uncertainty in Self-enforcing International Environmental Agreements. Journal of Environmental Economics and Management, 53, 6879.

Kotchen, M.J. (2005), Impure Public Goods and the Comparative Statics of Environmentally Friendly Consumption. Journal of Environmental Economics and Management, 49, 281-300.

Kotchen, M.J. (2006), Green Markets and Private Provision of Public Goods. Journal of Political Economy, 114, 816-834.

Kotchen, M.J. and Moore, M.R. (2007), Private Provision of Environmental Public Goods: Household Participation in Green-electricity Programs. Journal of Environmental Economics and Management, 53, 1-16.

Krupnick, A., Burtraw, D. and Markandya, A. (2000), The Ancillary Benefits and Costs of Climate Change Mitigation: A Conceptual Framework. In: OECD (ed.), Ancillary Benefits and Costs of Greenhouse Gas Mitigation, Paris, 53-93.

Markandya, A. and Rübbelke, D.T.G. (2004), Ancillary Benefits of Climate Policy. Jahrbücher für Nationalökonomie und Statistik, 224, 488-503.

Musgrave, R.A. (1959), The Theory of Public Finance. McGraw Hill, New York.

Pearce, D. (2000), Policy Framework for the Ancillary Benefits of Climate Change Policies. In: OECD (ed.), Ancillary Benefits and Costs of Greenhouse Gas Mitigation, Paris, 517-560.

Pittel, K. and Rübbelke, D.T.G. (2008), Climate Policy and Ancillary Benefits - A Survey and Integration into the Modelling of International Negotiations on Climate Change. Ecological Economics, forthcoming.

Rübbelke, D.T.G. (2002), International Climate Policy to Combat Global Warming An Analysis of the Ancillary Benefits of Reducing Carbon Emissions. Edward Elgar, Cheltenham.

Rübbelke, D.T.G. (2003), An Analysis of Differing Abatement Incentives. Resource and Energy Economics, 25, 269-295. 
Rubio, S. and Ulph, A. (2006), Self-enforcing International Environmental Agreements Revisited. Oxford Economic Papers, 58, 233-263.

Sandler, T. and Murdoch, J.C. (1990), Nash-Cournot or Lindahl Behavior? An Empirical Test for the NATO Allies. Quaterly Journal of Economics, 105, 875-894.

Smith, K.R. and Haigler, E. (2008), Co-Benefits of Climate Mitigation and Health Protection in Energy Systems: Scoping Methods. Annual Review of Public Health, 29, 11-25.

Ulph, A. (2004), Stable International Environmental Agreements with a Stock Pollutant, Uncertainty and Learning. Journal of Risk and Uncertainty, 29, 53-73.

Van Vuuren, D.P., Fengqi, Z., de Vries, B., Kejun, J., Graveland, C. and Yun, L. (2003), Energy and Emission Scenarios for China in the 21st Century - Exploration of Baseline Development and Mitigation Options. Energy Policy, 31, 369-387.

Vennemo, H., Aunan, K., Jinghua, F., Holtedahl, P., Tao, H. and Seip, H.M. (2006), Domestic Environmental Benefits of China's Energy-Related CDM Potential. Climatic Change, 75, 215-239. 


\section{Appendix}

For payoff function (22) in the text the first order condition of a non-signatory and signatory, respectively, are given by

(A1) $a b-b\left(Q^{N M}+Q^{M}\right)+\alpha\left(a b-b\left(\frac{Q^{M N}}{N-M}\right)\right)-c\left(\frac{Q^{M N}}{N-M}\right)=0$

(A2) $n\left(a b-b\left(Q^{N M}+Q^{M}\right)\right)+\alpha\left(a b-b\left(\frac{Q^{M}}{n}\right)\right)-c\left(\frac{Q^{M}}{n}\right)=0$

where we denote total abatement of non-signatories by $Q^{N M}$ and of signatories by $Q^{M}$ with the understanding that total abatement is $Q^{N M}+Q^{M}=(N-n) q_{i}^{N M}+n q_{i}^{M}$.

Solving (A1) and (A2) simultaneously delivers:

(A3) $Q^{M}=\frac{a b n\left(n c-b n \alpha N+b n^{2} \alpha+\alpha b N+\alpha^{2} b+a c\right)}{(\alpha b+c)\left(\alpha b+b n^{2}+b N-b n+c\right)}$

$$
Q^{N M}=\frac{a b(N-n)\left(\alpha^{2} b+\alpha b+b n^{2} \alpha-n \alpha b+\alpha c+c\right)}{(\alpha b+c)\left(\alpha b+b n^{2}+b N-b n+c\right)}
$$

Computing $q_{i}^{N M}=Q^{N M} /(N-n)$ and $q_{i}^{M}=Q^{M} / n$ we find

(A5) $\quad q_{i}^{M}-q_{i}^{N M}=\frac{a b(n-1)(c-\alpha b(N-1))}{(\alpha b+c)\left(\alpha b+b n^{2}+b N-b n+c\right)}$

which is only positive provided

(A6) $\quad c \geq \alpha b(N-1) \Leftrightarrow 1 \geq \alpha \gamma(N-1) \Leftrightarrow \frac{1}{(N-1) \gamma} \geq \alpha$ with $\gamma=b / c$. 
holds. It can be shown that condition (A6) is also sufficient that socially optimal abatement levels are weakly larger than those in the Nash equilibrium. Moreover, by substituting (A3) and (A4) into individual marginal benefits from global abatement, $a b-b\left(Q^{M N}+Q^{N}\right)$, it can also be shown that (A6) is a sufficient condition to ensure positive marginal benefits, i.e. $a b-b\left(Q^{M N}+Q^{N}\right) \geq 0$.

Casual observation of $Q^{N M}$ in (A4) reveals that $Q^{N M} \geq 0$ for any permissible assumption of parameters. This is less evident for $Q^{M}$ in (A3). First we notice that the denominator is always positive. The term in brackets in the numerator, taking note of $b / c=\gamma$, can be written as $n+\alpha+\alpha^{2} \gamma+\gamma \alpha\left(N+n^{2}-n N\right)$. Now $\left(N+n^{2}-n N\right)$ can be negative. Hence, we investigate $n+\gamma \alpha\left(N+n^{2}-n N\right)$ because $\alpha+\alpha^{2} \gamma$ is anyway positive. We replace $\alpha$ by its highest value given by (A6), namely $1 /((N-1) \gamma)=\alpha$ in $n+\gamma \alpha\left(N+n^{2}-n N\right)$. Then, we get after some manipulation $N+n^{2}-n>0$. Hence, we have proved that also signatories’ abatement is always positive.

In a next step we analyze the stability function $\Phi_{i}(n)=\pi_{i}^{M}(n)-\pi_{i}^{N M}(n-1)$. We find

$$
\Phi_{i}(n, \Gamma)=\pi_{i}^{M}(n)-\pi_{i}^{N M}(n-1)=\frac{(n-1)(\alpha b N-1-\alpha b)^{2}\left(b^{2} a^{2}\right) \cdot D}{E \cdot F \cdot G} \text { with }
$$$$
D=-\left(-3-4 b+n-2 b N+6 b n-2 b^{2} N n-4 b^{2} n^{2} N-3 b^{2} n^{2}+2 b^{2} n \alpha N+2 b^{2} n^{3} \alpha+b^{2} n \alpha^{2}\right.
$$$$
+2 n^{3} b^{2} N+b^{2} N^{2}+2 n b N+n b^{2} N^{2}+2 b n^{3}+b^{2} n^{5}+7 b^{2} n^{3}-5 b^{2} n^{4}-6 \alpha b-8 b n^{2}+2 n \alpha b
$$$$
\left.-8 b^{2} n^{2} \alpha-3 \alpha^{2} b^{2}-2 \alpha b^{2} N+6 \alpha b^{2} n-4 \alpha b^{2}\right)
$$$$
E=2\left(\alpha b+b n^{2}-3 b n+2 b+b N+1\right)^{2}, F=\left(\alpha b+b n^{2}+b N-b n+1\right)^{2}, G=(\alpha b+1)
$$

where $E, F$ and $G$ are positive and hence the numerator is positive. The first three terms in the denominator are also positive. Hence, the sign of $\Phi_{i}(n, \Gamma)$ depends on 
the sign of $D$. We observe that $D$ does not depend on the parameter $a$ and only depends on the relation $\gamma=b / c$. Hence the sign of $\Phi_{i}(n, \Gamma)$ and hence the sign of $D$ is a function of the parameters $\alpha, \gamma, N$ and $n$. Dividing $D$ by $c^{2}$ and using $\gamma=b / c$, we find

$$
\partial D / \partial N=-2\left(n \alpha \gamma+\gamma N+\gamma n^{3}-\alpha \gamma+\gamma N n-\gamma n-2 \gamma n^{2}-1\right)<0
$$

because the term in brackets is positive, noticing that $n \geq 1$. Moreover,

(A9) $\partial^{2} D / \partial N^{2}=-2 \gamma(\gamma+\gamma n)<0$

Substituting $N=2$ and $n=2$ into $D$ gives

(A10) $D(2,2)=1+4 \gamma+4 \alpha \gamma^{2}+\alpha^{2} \gamma^{2}+2 \alpha \gamma>0$

Substituting $N=3$ and $n=3$ into $D$ gives

(A11) $D(3,3)=-8 \gamma(9 \gamma+\alpha \gamma+1)<0$

and hence we can conclude that for $N \geq 3$ there is no stable coalition of more than 2 signatories. Hence, it remains to analyze stability for $n=2$ and $N \geq 3$.

Substituting $n=2$ into $D$ but making no assumptions about $N$ gives

(A12) $D(2, \Gamma)=A+B$ with

$$
A=1+\gamma(8-2 N)+\gamma^{2}\left(4 N+4-3 N^{2}\right) \text { and } B=\alpha \gamma^{2}(8-2 N)+2 \alpha \gamma+\alpha^{2} \gamma^{2} \text {. }
$$

We observe that $A$ and $B$ decrease in $N$ and for given $\alpha$ and $\gamma$ become negative for $N$ sufficiently large. Moreover, $B=0$ if $\alpha=0$. Solving $D(2, \Gamma)$ for $\gamma$, setting $\alpha=0$, gives 
(A13) $\gamma_{1}=\frac{4-N+\sqrt{3-3 N+N^{2}}}{3 N^{2}-4 N-4}$ and $\gamma_{2}=\frac{4-N+\sqrt{3-3 N+N^{2}}}{3 N^{2}-4 N-4}$

As it can be shown, only the first solution is positive for $N \geq 3$ and hence the relevant one, which we denote by $\bar{\gamma}(N)$ in the text. Since $A$ is concave in $\gamma$ for $N \geq 3$, we can conclude that $\Phi_{i}(2, \Gamma) \geq 0$ if $0 \leq \gamma \leq \bar{\gamma}(N)$. It is straightforward to check that $\bar{\gamma}(N)<1$ and $\partial \bar{\gamma}(N) / \partial N<0$ for $N \geq 3$.

Investigating $B$ and computing $\partial B / \partial \alpha=2 \gamma+\gamma^{2}(8-2 N+2 \alpha)$ reveals that $\alpha$ has clearly a positive influence on stability for $N \in\{3,4\}$. For $N \geq 5, \alpha$ must be sufficiently large. Noticing that $B$, and hence $A+B$, is concave in $\alpha$ $\left(\partial B^{2} / \partial \alpha^{2}=2 \gamma^{2}>0\right)$, we solve $D(2, \Gamma)$ for $\alpha$ and find

(A14) $\alpha_{1}=\frac{-1+\gamma N-4 \gamma+2 \sqrt{\gamma^{2} N^{2}-3 \gamma^{2} N+3 \gamma^{2}}}{\gamma}$ and

$$
\alpha_{2}=\frac{-1+\gamma N-4 \gamma-2 \sqrt{\gamma^{2} N^{2}-3 \gamma^{2} N+3 \gamma^{2}}}{\gamma}
$$

where only the first solution is positive and hence the relevant one. Hence, observing (A6), we get

$$
\frac{1}{(N-1) \gamma} \geq \alpha \geq \frac{-1+\gamma N-4 \gamma+2 \sqrt{\gamma^{2} N^{2}-3 \gamma^{2} N+3 \gamma^{2}}}{\gamma}=\Psi(\gamma, N)
$$

Obviously, $1 /((N-1) \gamma)$ decreases in $N$ and $\gamma$. For $\Psi(\gamma, N)$ we find

$$
\text { (A16) } \frac{\partial \Psi(\gamma, N)}{\partial N}=\frac{\sqrt{\gamma^{2}\left(3-3 N+N^{2}\right.}+2 \gamma N-3 \gamma}{\sqrt{\gamma^{2}\left(3-3 N+N^{2}\right.}}>0 \text { and } \frac{\partial \Psi(\gamma, N)}{\partial \gamma}=\frac{1}{\gamma^{2}}>0
$$


recalling that we assume $N \geq 3$. Hence, with increasing $N$, the range of $\alpha$ that satisfies (A15) decreases. Moreover, the possibility of increasing $\gamma$ above $\bar{\gamma}(N)$ to $\bar{\gamma}(N, \alpha)$ is also limited. For instance, using (A13) and (A15), we find:

\begin{tabular}{|l|l|l|l|l|l|l|l|l|}
\hline $\mathrm{N}$ & 3 & 4 & 5 & 6 & 7 & 8 & 9 & 10 \\
\hline $\bar{\gamma}(N)$ & 0.41 & 0.19 & 0.12 & 0.09 & 0.07 & 0.06 & 0.05 & 0.04 \\
\hline $\bar{\gamma}(N, \alpha)$ & 0.60 & 0.25 & 0.15 & 0.11 & 0.08 & 0.07 & 0.06 & 0.05 \\
\hline
\end{tabular}

Inserting $\bar{\gamma}(n)$ for $\gamma$ in (A15) shows however that $\frac{1}{(N-1) \gamma}-\Psi(\gamma, N)>0$ for $N \geq 2$ and hence there always exists an $\alpha$ that satisfies condition (A15) at the limit of $\gamma=\bar{\gamma}(n)$.

Investigating the closing the gap index as defined in (10) in the text for $n=2$, we find

(A17) $\quad C G I=\frac{2\left(\alpha \gamma+\gamma N^{2}+1\right) \cdot H}{N(\alpha \gamma+2 \gamma+\gamma N+1)^{2}(\alpha \gamma+1)(N-1)^{2}}$ with

$H=-3 \alpha^{2} \gamma^{2}+2 \alpha \gamma^{2} N+2 \alpha \gamma^{2} N^{2}-2 \alpha \gamma^{2} N+4 \alpha \gamma N-6 \alpha \gamma+2 \gamma^{2} N-\gamma^{2} N^{2}+2 \gamma N^{2}-2 \gamma N+2 N-3$

Differentiating CGI with respect to $\alpha$ gives

(A18) $\frac{\partial C G I}{\partial \alpha}=-\frac{2 \gamma^{2}(N-2)(\alpha \gamma+\gamma N+1) \cdot J}{N(\alpha \gamma+2 \gamma+\gamma N+1)^{3}(\alpha \gamma+1)^{2}(N-1)^{2}}$ with $J=I+K$,

$I=\left(2 N^{2}-N-6\right)-2 \gamma\left(N^{2}-N\right)-\gamma^{2}\left(N^{3}+N^{2}\right)$ and

$K=\alpha \gamma\left(4 N^{2}-2 N-12\right)+\alpha^{2} \gamma^{2}\left(2 N^{2}-N-6\right)-2 \alpha \gamma^{2}\left(N^{2}-N\right)$.

We want to show that $J>0$ and hence this derivative is negative. First we concentrate on $I$. We notice that $I$ is concave in $\gamma$. Setting $I=0$ and solving for $\gamma$ gives two values of which only one is positive. This value, denoted by $\gamma_{\omega}(N)$, is given by 
(A19) $\gamma_{\omega}(N)=\frac{-N+1+\sqrt{2 N^{2}-9 N-5+2 N^{3}}}{N(N+1)}$.

Thus, $I \geq 0$ if $\gamma \leq \gamma_{\omega}(N)$. Now it can be shown that $\gamma_{\omega}(N)>\bar{\gamma}(N)$ for $N \geq 4$ and hence $I>0$ for $N \geq 4$ and $0 \leq \gamma \leq \bar{\gamma}(N)$, i.e. the parameter range for which a coalition of two players is stable without ancillary benefits. We now turn to $K$ and find:

$$
\begin{aligned}
& \frac{\partial K}{\partial \alpha}=\gamma\left(4 N^{2}-2 N-12\right)+2 \alpha \gamma^{2}\left(2 N^{2}-N-6\right)-2 \gamma^{2}\left(N^{2}-N\right) \text { and } \\
& \frac{\partial^{2} K}{\partial \alpha^{2}}=2 \gamma^{2}\left(2 N^{2}-N-6\right)
\end{aligned}
$$

The second derivative is positive for $N \geq 3$ and hence $K$ is convex in $\alpha$. Hence, setting the first derivative to zero and solving for $\alpha$ gives us a minimum. We find:

(A21) $\alpha^{*}=\frac{2\left(N^{2}-N\right)-\frac{1}{\gamma}\left(4 N^{2}-2 N-12\right)}{2\left(2 N^{2}-N-6\right)}$.

Now the numerator can only be positive if $1 / \gamma$ is sufficiently small. Since we consider the range $0 \leq \gamma \leq \bar{\gamma}(N)<1$, we test whether the numerator of $\alpha^{*}$ is positive for $\bar{\gamma}(N)$. It turns out that this is not the case for $N \geq 3$. Consequently, $K$ is minimized for $\alpha=0$. Then, however, $K=0$, and hence $J>0$ and therefore $\partial C G I / \partial \alpha<0$ for $0 \leq \gamma \leq \bar{\gamma}(N)$. Consequently, we can conclude that $\alpha$ can only have a positive influence on CGI if and only if $\gamma>\bar{\gamma}(N)$ because then no coalition is stable without ancillary benefits. 